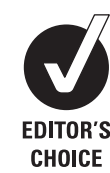

$10.1136 / \mathrm{ebm} .2011 .100101$

${ }^{1}$ Cooperative Studies Program, VA Connecticut Healthcare System, West Haven, Connecticut, USA

${ }^{2}$ Internal Medicine, Yale University School of Medicine, West Haven, Connecticut, USA

Correspondence to:

John Concato

Clinical Epidemiology Research

Program, VA Connecticut

Healthcare System, 950

Campbell Avenue, CERC 151B,

West Haven, CT 06516, USA;

john.concato@yale.edu

Randomised controlled trial

\title{
Randomised trial of radical prostatectomy versus watchful waiting finds reduced risk for death but uncertainty still reigns
}

\section{John Concato, ${ }^{1,2}$ Peter Guarino ${ }^{1}$}

\author{
Commentary on: Bill-Axelson A, Holmberg L, Ruutu M, et al. Radical prostatectomy versus watchful \\ waiting in early prostate cancer. N Engl J Med 2011;364:1708-17.
}

\section{Context}

Data comparing surgery versus watchful waiting for prostate cancer are important, and the results are also relevant when evaluating whether and when screening for prostate cancer is effective. ${ }^{1}$ Thus, deciding whether such study results are valid, and how well they generalise, is a prominent challenge in men's health.

\section{Methods}

This randomised trial seeks to determine whether mortality from prostate cancer is lower after radical prostatectomy than with primary expectant management. As an ongoing project for more than 20 years, prior major reports appeared in $2002,{ }^{2} 2005^{3}$ and $2008 .^{4}$ The current report extends follow-up by 3 years and focuses on low-risk prostate cancer (Gleason histological score $<7$, prostate-specific antigen level $<10 \mathrm{ng} / \mathrm{ml}$ ) and younger men ( $<65$ years of age).

\section{Findings}

In summary, 695 men with localised and well-differentiated or moderately differentiated prostate cancer (mean age 65 years) were followed for a median 12.8 years; more than half the study population had died, with almost two-thirds of deaths attributed to conditions other than prostate cancer. The cumulative incidence of death from prostate cancer at 15 years was $14.6 \%$ for men randomised to surgery and $20.7 \%$ for men randomised to watchful waiting; this difference is clinically important and statistically significant $(\mathrm{p}=0.01)$.

\section{Commentary}

Among technical issues, randomisation was centralised, informed consent used the Zelen approach ${ }^{5}$ and the trial was not blinded (as is common for surgical trials). Nonsurgical management can vary from watchful waiting, as in this study, to intense, active medical surveillance. In addition, surgical and non-surgical care are both susceptible to geographical differences and trends over time (eg, nerve-sparing surgery, androgen deprivation). Thus, applying the study results to contemporary patients requires an assessment of changing clinical practice.
Selecting all-cause mortality, prostate-cancer mortality, and metastases as outcomes is laudable, and the data were analysed appropriately based on competing risks. The study abstract states that the survival benefit of surgery was limited to younger men and was also seen among men with low-risk prostate cancer. Interpreting the findings from subgroup analyses (based on age and risk, for multiple outcomes) should be done with caution, ${ }^{6}$ however, as should making multiple inferences from the same study on multiple occasions.

A major concern is how the study population compares to current clinical practice. More importantly, and overshadowing concern regarding crossovers and changes over time in Gleason scoring, only 5\% of the participants were detected by screening in this trial, whereas the vast majority of prostate cancer cases in men are screen-detected in the USA (and now in most countries). Assessing generalisability is intended to address this problem, but details of a study population tend to be forgotten after a trial is published and deemed valid. A call for considering validity and generalisability together determining whether the results are 'accurate' for those who would receive the intervention - seems especially needed in this context. ${ }^{7}$

This Scandinavian study represents a landmark clinical trial in urology, but the authors were careful to "caution that [the] low-risk group cannot be compared directly with men who are currently included in active-surveillance protocols because few of the men in [the] low-risk group had a tumor that was detected by means of a screening test." Other trials with a similar focus are ongoing in the USA and UK, and the recent experience ${ }^{18}$ with trials of screening for prostate cancer may be repeated - knowledge is advanced after trials are published, but uncertainty is not eliminated or reduced as much as anticipated (or desired). For the foreseeable future, to guide therapy in prostate cancer, physicians should continue to use their professional judgement, informed by available evidence and combined with patient preference.

Competing interests None. 


\section{References}

1. Concato J. What will the emperor say? Screening for prostate cancer as of 2008. Cancer J 2009;15:7-12.

2. Holmberg L, Bill-Axelson A, Helgesen F, et al. A randomized trial comparing radical prostatectomy with watchful waiting in early prostate cancer. $N$ Engl J Med 2002;347:781-9.

3. Bill-Axelson A, Holmberg L, Ruutu M, et al. Radical prostatectomy versus watchful waiting in early prostate cancer. N Engl J Med 2005;352:1977-84.

4. Bill-Axelson A, Holmberg L, Filén F, et al. Radical prostatectomy versus watchful waiting in localized prostate cancer: the Scandinavian prostate cancer group-4 randomized

trial. J Natl Cancer Inst 2008;100:1144-54.

5. Zelen M. A new design for randomized clinical trials. N Engl J Med 1979;300:1242-5.

6. Sun X, Briel M, Walter SD, et al. Is a subgroup effect believable? Updating criteria to evaluate the credibility of subgroup analyses. BMJ 2010;340:c117.

7. Concato J, Horwitz RI. Beyond randomised versus observational studies. Lancet 2004;363:1660-1.

8. Barry MJ. Screening for prostate cancer - the controversy that refuses to die. N Engl J Med 2009;360:1351-4. 